\title{
Design and Development of Generators-Power Grid Dynamic Performance Online Monitoring System Based on WAMS data
}

\author{
Xiu-ping $\mathrm{SHI}^{1 *}$, Wei Tang ${ }^{2}$,Jun $\mathrm{PU}^{3}$, Yuan-chao HUANG ${ }^{1}$, Xin-qiao FAN ${ }^{1}$ and \\ Wen-chao $\mathrm{ZHANG}^{1}$
}

1. NARI Group Corporation, Nanjing, 210003, China

2. Anhui Electric Power Company of SGCC,Hefei, 230001, China

3. CEPRI, Beijing, 100192, China

Key words:WAMS, Generator,Control System, Online Monitoring.

\begin{abstract}
Generator set and its control system are the most important dynamic components in power system. It has a vital effect on the safety and stability of power system. In order to ensure the safe and stable operation of power system, we must strengthen the monitoring of power generation units and control system. This paper introduces the hardware structure and software structure of Generators-Power Grid Dynamic Performance Online Monitoring System based on WAMS data, and the main functions and performance.The practical operation of system in the provincial power grid networkis introduced in this paper, which fully demonstrates the practicability and accuracy of the system, the operation of the system provides the powerful tool and means for the safety and stability of the power grid.
\end{abstract}

\section{Introduction}

Generator set and its control system are the most important dynamic components of power system, having a crucial impact on stability and safety of power system. With the development of China power grid, a rapid increase in grid-scale, sources type and number can be seen and power system dynamic characteristics is becoming more complicated.In order to guarantee the safe and stable operation of power system, it's necessary to focus more on power generation units and control system monitoring. For conventional hydraulic or thermal engine group, the main task is to conduct a comprehensive monitoring on the key variables of generator excitation and governor control system, giving a full play to its continuous and controllable characteristics, which will play a supporting role to power system stability and safety.

The widespread deployment of WAMS provides a strong supportfor the monitoring of generator and its control system.By connectingcritical internal variables of the conventional generator set control systeminto WAMS system, comprehensive monitoring of the conventional unit control performance can be achieved and fast and accurate recognition can be conducted as soon as power grid disturbance or oscillation occurs, then we can evaluate the performance of generation units in power systemdepending on its response todisturbance.

In view ofpower network situation, the proposedsource network dynamic performance monitoring and evaluation onlinesystem based on the WAMS information can make full use of the real-time data from WAMS,analyzing the unit control system internal key variablesin-depth, detecting power grid disturbanceand oscillation effectively and quickly, identifyingthe abnormal running state of unit, examiningwhetherthe response of unitto disturbance and oscillatory meet the requirements of power grid or not, evaluatingthe dynamic performance of generator set as well as providing technical support for the safety and stability of power grid.

\section{System Design}

The application subsystem of the D5000 platform is based on the source network dynamic performance onlinemonitoring and evaluation system, which aims toachieve generator condition online monitoring,network generation unitperformance online monitoring and assessment, 
database services, human-computer interface display function, generator set dynamic performance online monitoring and evaluation function.

\subsection{System hardware structure}

The system hardware structure mainly includes data server, on-line application server, advanced application function display workstation, maintenance work station etc., system platform architecture diagram is shown in figure 1.

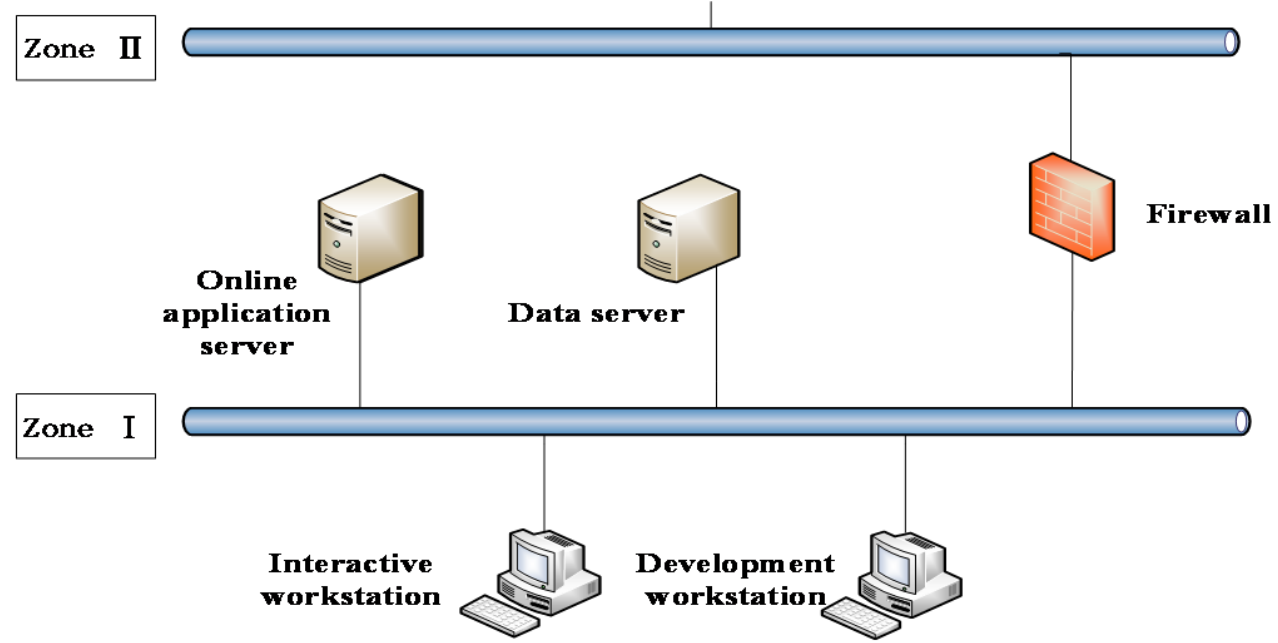

Figure 1.Architecture diagram of the system platform

\subsection{System software structure}

\subsubsection{Software service configuration}

Basic services that system software platform provides including real-time library services, historical library services, event library service, file service aiming for providing related services for dataapplication system and software. The services and programs which are deployed and configured on each node are shown in table1.

Table 1. Node service configuration

\begin{tabular}{|c|c|c|}
\hline & & Safety zone I \\
\hline $\begin{array}{l}\text { Nodes } \\
\text { Number }\end{array}$ & Node machine & Services and programs deployment \\
\hline \multirow{12}{*}{4} & \multirow{4}{*}{$\begin{array}{l}\text { Online } \\
\text { application server }\end{array}$} & Generator set operation performance online monitoring \\
\hline & & $\begin{array}{l}\text { Excitation system performance online monitoring and } \\
\text { evaluation }\end{array}$ \\
\hline & & $\begin{array}{l}\text { Speed control system performance online monitoring and } \\
\text { assessment services }\end{array}$ \\
\hline & & $\begin{array}{c}\text { Real-time D5000 database \&Time series database } \\
\text { services }\end{array}$ \\
\hline & \multirow[b]{2}{*}{ Data server } & Disturbance data service \\
\hline & & $\begin{array}{c}\text { Real-time D5000 database \& Time series database } \\
\text { services }\end{array}$ \\
\hline & \multirow{3}{*}{$\begin{array}{c}\text { Interface } \\
\text { workstation }\end{array}$} & $\begin{array}{c}\text { Interface program of source network dynamic } \\
\text { performance online monitoring and evaluation system }\end{array}$ \\
\hline & & D5000 database services \\
\hline & & Off-line auxiliary analysis tool \\
\hline & \multirow{3}{*}{$\begin{array}{c}\text { Maintenance and } \\
\text { development } \\
\text { work station }\end{array}$} & D5000 database services \\
\hline & & System configuration utilization program \\
\hline & & Qt/other applications development tools \\
\hline
\end{tabular}




\subsubsection{Software functions structure}

Software functions mainly include data fusion and dynamic monitoring, AVR and PSS performance evaluation, governor performance evaluation, AGC and AVC performance evaluation, generator dynamic performance evaluation and other functional modules and software functions structure is as shown in figure 2 .

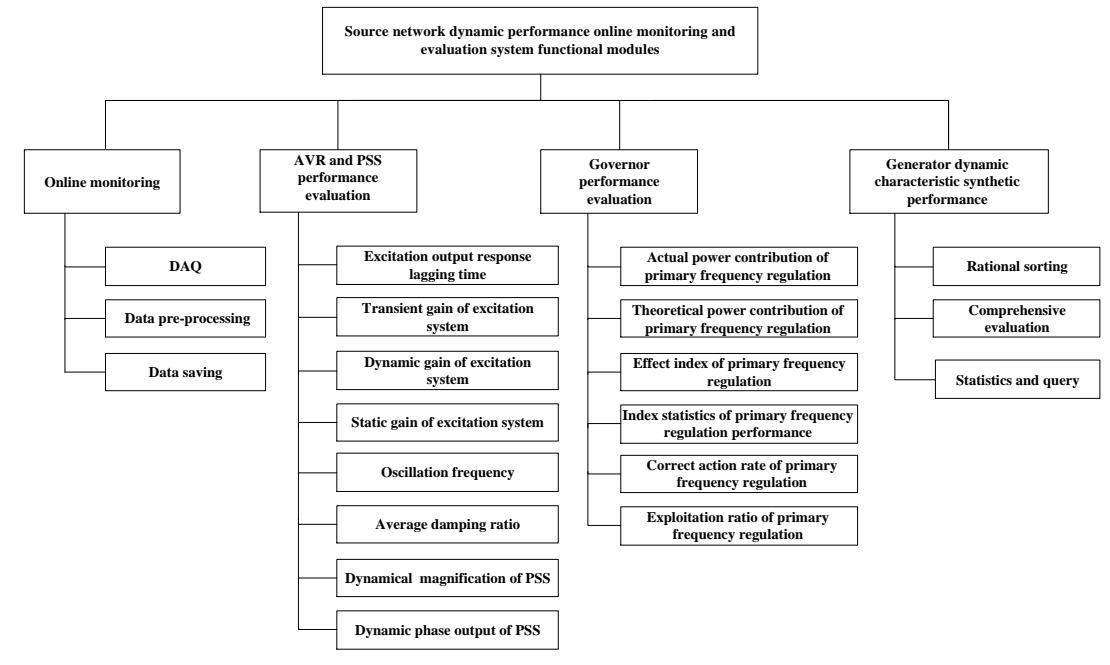

Figure 2.Software architecture diagram

\section{System function and performance}

\subsection{Data fusion and dynamic monitoring modules}

By building up data interface with D5000 system or PSASP we acquired model parameters and operation data in order to achieve the function of data fusion. For the uneven quality of different types of real-time data in distribution network, the data to be analyzed should be pro-processed. The data quality should be evaluated and unqualified data should be eliminated to avoid the interference of bad data to monitoring analysis, realizing the automatic verification of various types of data.

\subsection{AVR and PSS performance evaluation}

The functional module monitors and evaluates the dynamic performance of excitation system, usingthe actual response data of different units afterpower grid disturbance, combining the basic parameters of the excitation system settings, such asmagnification and excitation limits, to calculate the per unit value of units' controlled volumes and controlled variables. For the effective voltage disturbance, it would extract excitation output response lagging time, transient magnification of excitation system, dynamic magnification ratio, staticmagnification, etc. to evaluate the performance of generator excitation system. For grid oscillation, the system extracts oscillation frequency, oscillation damping ratio, PSS dynamic magnification, PSS dynamic phase output and other indexes to evaluate the performance of generator excitation system.

\subsection{Governor performance monitoring module}

This modulemonitors and evaluates the governor dynamic characteristics, basing on units' speed regulationsystem instruction, speed deviation, power deviation and other responses, combining the basic operation mode and parameter setting ofspeed regulation system, to derive the per unit value of units' controlled volumes and controlled variables. The system extracts actual power contribution, theoretical power contribution, effect index, index statistics of performance, correct action rate, exploitation ratio, etc. of the primary frequency regulation. 


\subsection{AGC/AVC performance monitoring module}

This module makes use of AGC/AVC monitoring data to record AGC/AVC commissioning and ceasing data as well as their action situation and then calculate AGC/AVC regulation performance index.

AGC performance index includes:

(1) AGC Availability ratio

(2) AGC regulation performance

1) Regulation rate

2) Regulation precision

3) Response time

(3) AGC regulation performance comprehensive index

AVC performance index includes:

(1) Units' AVC commissioning rate

(2) Units' AVC regulation pass rate

(3) Bus bar voltage pass rate

\subsection{Unit dynamic characteristics evaluation module}

(1) Propose rational sorting algorithm taking disturbance difference

The response of unit control system to disturbance will vary with the situation changes, which will influence the evaluation rationality of unit dynamic characteristic.

1) Analysis effects that different disturbance scenarios would have on dynamic performance index of generators;

2) Compare and manage the different dynamic performances of unit.

(2) Comprehensive evaluation of unit characteristic

1) quantify the importance of each single dynamic performance index and derive the weight of each single performance index of unit.

2) Obtainedthe comprehensive evaluation of the dynamic performance of the generator according to the synthesis of several performance indexes of the unit.

(3) Statistics and query

Display the results of unit dynamic characteristic evaluation, taking the results as the guideline of system operation.

1) Tally, query and analyzethecalculationresults according to different indicatorsmonthly, quarterly and yearly.

2) Automaticallygenerateunit dynamic performance index reportmonthly, quarterly, yearly orin custom date range.

3) Automaticallygeneratesynthesis evaluation of generator dynamic characteristic report.

\section{System application}

\subsection{Excitation system performance evaluation}

After the excitation system performance online monitoring and evaluation module putting into operation, several unconventional dynamic processes weredetected. By processing and analyzing the data of excitation system, the performance monitoring and evaluation can be achieved based on the response characteristics.

(1) On January 6, 2016, 04:34:58, $440 \# 1$ generatoroccurred voltage sag, as shown in figure 3. The system detected this event accurately and fast, thenit finished online evaluation.And the result shows that the dynamic response performance of excitation system operates well. 


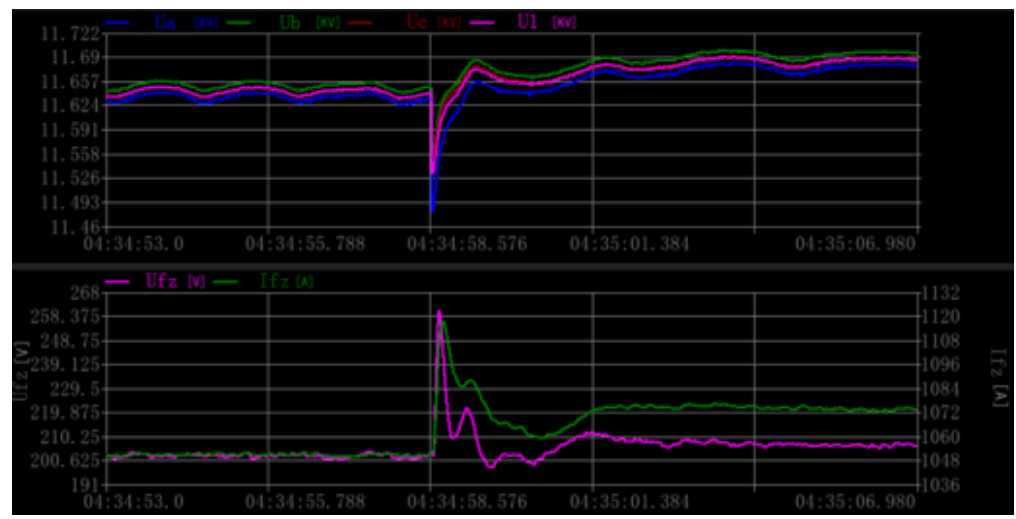

Figure 3. Dynamic response process of voltage sag

(2) In September 24, 2015, 11:55:00, the 160 electric power plant's \#5 generator shut down and the Dynamic Performance Online Monitoring System accurately monitored the accident. Meanwhile, the system made online analysis and evaluation. Based on the analysis result, we can found that the generator set's excitation system had good dynamic performance during the process of shutting down. As shown in figure 4.

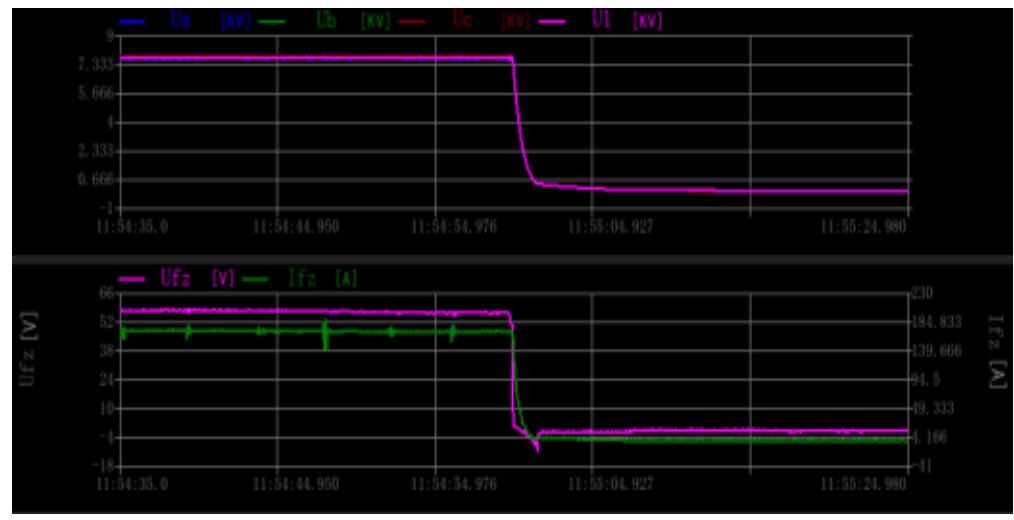

Figure 4. Dynamic response process of down

\subsection{Application of the governor characteristics evaluation module}

After the online governor dynamic characteristics monitor and evaluation module putting into operation, it detected the process of power system frequency crossing the dead zone many times. Based on the processing and analysis of governor's dynamic process data, the module succeeded to calculate the parameter of governor's characteristics, realizing the function to monitor and evaluate the governor's characteristics.

(1) In December 23, 2015, 16:01:10, the 840 electric power plant's \#1 generator's frequency fluctuated then primary frequency modulation moduleworked. The online governor dynamic characteristics monitor and evaluation module accurately recorded the whole process of primary frequency modulation. Meanwhile, it analyzed and evaluated the process. Based on the result, we found that the governor's dynamic response performance is well. Shown as figure 5 . 


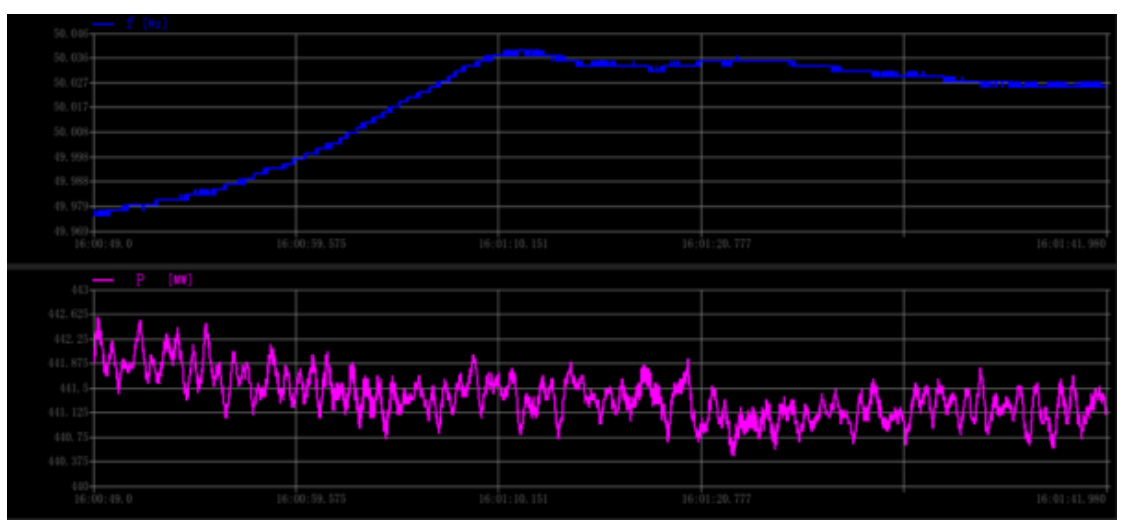

Figure 5. Operation process of primary frequency regulation

\section{Conclusion and discussion}

Generators-Power Grid Dynamic Performance Online Monitoring System Based on WAMS data has been put into operation in many provincial power grid, the system can quickly detect all kinds of disturbance, oscillation in network and it can also identify the abnormal operation of the unit online. By calculating and analyzing key variables of unit internalcontrol system,we can evaluate network dynamic performance online, and assess whether the response of the unit in disturbance and oscillation conform to the requirements of the grid based on evaluation results; The system can monitor and evaluate of the dynamic performance of grid connected power plants at all levels online, providing a powerful technical guarantee for the safe and stable operation of the power grid.

\section{References:}

[1] Lu Jinjun, Dai Zemei. Zhang Li,"Power system monitoring and evaluation based on WAMS,"Automation of Electric Power Systems, 2012, 36(8).

[2] Huang Hongyang, XuZheng, "Generator excitation control scheme based on wide area measurement system," Power System Protection and Control 2013, (6).

[3] Xiong Xiaoping, "Power system WAMS reliability analysis and optimal design," Automation of Electric Power Systems2013.

[4] MengFanch,DongXiaoliang, GaoZhiqiang, Li Guoqing, XieXiaorong, "PSS Online Assessment based on WAMS/PMU," Electric Power Automation Equipment 2012, 32(10).

[5] Li Qian, Xu Yong, Zhang Daonong. A statistical evaluation method of primary frequency regulation of power plant based on WAMS information filtering AGC Effect[M]. CSEE 2014 Annual Conference. 